\section{Induced hypothermia after cardiopulmonary resuscitation: possible adverse effects}

RUDOLF MILANOVIC

Department of Surgery,

University Hospital Dubrava Zagreb

SANJA HUSEDZINOVIC • NIKOLA BRADIC $(\square)$

Department of anesthesiology, reanimatology and intensive

care medicine,

University Hospital Dubrava, Zagreb

Gojko Susak Avenue No 6 10000 Zagreb, Croatia

Phone: + 38512903197

Fax: + 38512903440

E-mail: nbradic@kbd.hr

\begin{abstract}
The last several years have seen an increased interest in the use of induced hypothermia after witnessed cardiopulmonary resuscitation (CPR). The main reason for its use is protection of the brain and hence, better neurological outcome in these patients. Therefore, induced hypothermia after CPR has become a part of standard recommendations in the 2005 Resuscitation Guidelines. At the same time, hypothermia can have many adverse effects. In the event of pre-hospital and/or in-hospital induction of hypothermia, without adequate monitoring and controlled cooling, hypothermia can cause serious complications, without beneficial effects on the brain. This article explains the most frequent adverse effects of hypothermia and possible hazardous outcomes for patients.
\end{abstract}

Key words: cardiopulmonary resuscitation, hypothermia, hemodynamics

\section{Introduction}

Interest in hypothermia for neuroprotection began in the 1980s. For some surgical procedures, primary cardiac and neurosurgical, hypothermia can be used for conservation of brain tissue and decreasing cerebral metabolic oxygen rate $\left(\mathrm{CMRO}_{2}\right)$. States of strongly controlled and deep hypothermia are usually achieved using cardiopulmonary bypass devices and neuroprotective agents combined with brain monitoring. The last several years have seen an increased interest in the use of induced hypothermia after witnessed cardiopulmonary resuscitation (CPR). The main reason for its use is to protect the brain against irreversible hypoxic damage and hence, to achieve better neurological outcomes in these patients. For these reasons, induced hypothermia after CPR has became a part of the 2005 Guidelines for Resuscitation (1)

\section{Clinical purpose}

By definition, hypothermia is a body temperature less than $36^{\circ} \mathrm{C}$ and it is divided into three stages: mild hypothermia, when the body temperature is between $35^{\circ} \mathrm{C}-32^{\circ} \mathrm{C}$, moderate hypothermia when the body temperature is between $32^{\circ} \mathrm{C}$ and $30^{\circ} \mathrm{C}$, and deep hypothermia when the body temperature is less than $30^{\circ} \mathrm{C}$. Methods for decreasing core temperature have varied. In animal studies, cardiopulmonary bypass, cold aortic flush, external cooling and peritoneal cooling are some examples of the methods that have been used. Clinical studies have been performed using external cooling with icepacks, forced cooled air or a cooling helmet device or by using a rapid (30 min) intravenous infusion of a large volume $(30 \mathrm{ml} / \mathrm{kg})$ of lactated Ringer's solution, cooled at $4^{\circ} \mathrm{C}$. The main problem with all of these methods is the slow decrease in core temperature. The measured drop in temperature was between $0.3^{\circ} \mathrm{C} / \mathrm{h}$ for forced cooled air (2), $0.9^{\circ} \mathrm{C} / \mathrm{h}$ for icepacks (3), and the highest decrease in core temperature $\left(1.7^{\circ} \mathrm{C}\right)$ was reached with fluid administration (3). All of the above mentioned methods do not provide a quick and safe means of decreasing core temperature. In pre-hospital and/or in-hospital induction of hypothermia, without adequate monitoring and controlled cooling, hypothermia can cause serious complications, without beneficial effects on the brain.

\section{Adverse effects of hypo- thermia on the heart and hemodynamics}

The adverse effects of hypothermia mainly disturb heart function and func- 
tion of the vascular system. It is known that more than $80 \%$ of all cardiac arrests are caused by presumed cardiac disease, of which more than $60 \%$ of adult deaths result from coronary heart disease (4). According to these data, cardiac and hemodynamic disturbances are augmented by hypothermia. Firstly, after return of spontaneous circulation (ROSC), following ventricular fibrillation, the myocardium shows huge electrical and mechanical instability. This instability may cause refibrillation within a short time after ROSC. Refibrillation is especially dangerous if cooling starts immediately after ROSC and if performed by emergency medical personnel, as reported by Nolan et al (5), and Bernard et al (3). In comparison with normothermic myocardium, the fibrillating hypothermic myocardium is characterized by increased contraction amplitude (6), decreased contraction velocity (6) and a decreased median fibrillation frequency (7). These characteristics of the fibrillating hypothermic heart may contribute to a reduced efficiency of electrical counter shock therapy, as observed in both experimental animals and humans (810). Electrophysiological changes associated with ischemia are similar to those induced by hypothermia (11). As mentioned earlier, most cardiac arrests are a consequence of myocardial ischemia. Thus ischemia may potentiate the arrhythmogenic potential of hypothermia (11). Improving myocardial perfusion by increasing coronary perfusion pressure (CPP) may reverse some of the electrophysiological changes and improve the outcome of CPR. This increase in CPP was associated with a significant improvement in defibrillation success (12). To improve CPP it is necessary to involve vasoactive drugs. In a state of post CPR instability, these agents can also provoke malignant arrhythmias. These agents increase myocardial oxygen consumption, what in a state of compromised coronary circulation, can worsen myocardial ischemia and enlarge the infarction zone. On the other hand, it is known that receptors for inotropes and vasoactive drugs become unresponsive during hypothermia and that the action of these drugs shifts from the heart to the vasculature producing an undesirable increase in peripheral vascular resistance.

In one of the first studies about induced hypothermia, Bernard et al (3) had significantly higher values of systemic vascular resistance and lower values of cardiac index in a group of patients with hypothermia throughout the whole 24 hour period of treatment compared with patients in normothermia. This unfavourable effect was not commented on and outcome, according to their cardiac function after induced hypothermia, was not evaluated. Furthermore, in the same study, patients with induced hypothermia received much more epinephrine infusions.

In a newer study by Kliegel et al (13), was instilled $2000 \mathrm{~mL}$ of ice-cold infusions cooled to $4^{\circ} \mathrm{C}$ via an endovascular catheter. The only hemodynamic monitoring was invasive arterial pressure monitoring. Except for 3 patients, all other patients had a cardiac event due to acute coronary syndrome (ACS). Unfortunately, there is no data on central venous pressures (CVP) and/or changes in pulmonary artery pressures during the cooling process with high doses of infusions. During ACS, especially with acute left ventricular myocardial infarction, the main problem is decreasing left ventricular compliance and diastolic dysfunction. These patients are very vulnerable to volume overload and rapidly develop pulmonary edema, which happened in this study in $7 \%$ of patients. There is no evidence in this study as to the reasons for pulmonary edema in these patients.

\section{Adverse effects of hypo- thermia on other organs and organ systems}

There are many adverse effects of hypothermia on the functioning of organs and organ systems. Firstly, hypothermia impairs immune function and leads to nosocomial pneumonia. Secondly, hypothermic patients with a core temperature $1.5^{\circ} \mathrm{C}-2.0^{\circ} \mathrm{C}$ below normal $\left(35.5^{\circ} \mathrm{C}-\right.$ $35.0^{\circ} \mathrm{C}$ ) have an approximately $19 \%$ rate of infection as opposed to a $6 \%$ rate of infection in normothermic patients. These findings may be a result of the fact that hypothermia increases a patients' vulnerability caused by vasoconstriction and impaired immunity. In a large European study (2) the incidence of sepsis was almost twofold higher in the group of patients randomised to hypothermia than in normothermic patients (7\% vs. $13 \%$ ).

The risk of infection can be augmented by poor glycemic control. An important side effect is insulin resistance and decreased insulin release. Other adverse effects in the gastrointestinal system are decreased gastrointestinal motility, raised serum amylase and liver enzymes which can produce metabolic acidosis as a result of increased lactate concentrations and increased production of free fatty acids, ketones and glycerol.

Intracellular movements of potassium, magnesium, and phosphate during hypothermia lead to lowered serum concentrations of these anions and may produce serious arrhythmias.

Hypothermia has several negative effects on the hematological system. Bleeding time is lengthened as a result of reduction in the number and function of platelets sequestered by the spleen and liver. The coagulation cascade may also be impaired, white cell count also decreases and their movement is also impaired.

Hypothermia produces significant altered actions of many drugs commonly used during the post-resuscitation period. The action of many agents used in CPR may be ineffective or occur with delayed action. Lidocaine has no documented beneficial effects during hypothermia. Clinical data suggest that amiodarone has an immediate antiarrhythmic effect, present already a few minutes after intravenous bolus administration $(14,15)$, but in the state of hypothermia, amiodarone had no beneficial effects on the resuscibilty of the fibrillating hypothermic heart (12). In a state of hypothermia, non-depolarizing muscle relaxants with a predictable half-life, prolong their average duration of action more than double, with an unpredictable time of action. 


\section{Conclusion}

The main reason why inducing hypothermia is now included in the new recommendations for CPR is to decrease the risk of hypoxic brain damage. Unfortunately, hypothermia may often cause very serious adverse effects or complications, which can be hazardous for the patient.

Many studies today, looking at induced hypothermia after CPR, are orientated exclusively on the neurological outcome of patients. In many of these studies there is no, or only very weak, evidence concerning functions and adverse effects of hypothermia on other organs or organ systems. Clinical follow-up of primary heart functioning after arrest is not well documented. Changes in hemodynamics during the first 24 hours after ACS may produce deleterious conditions for the patients, especially if relatively uncontrolled measures of cooling are used. Induction of core hypothermia using surface cooling blankets is a relatively slow process, especially when patients are vasoconstricted $(16,17)$. On the other hand, the use of large amounts of ice-cold $\left(4^{\circ} \mathrm{C}\right)$ volume can decrease core temperature rapidly, but may be very hazardous in patients with unstable heart function and rhythm, and, finally, may cause depletion of platelets, leukocytes and impair coagulation function.
From all the studies, it is not clear in which real time it is best to perform induced hypothermia to achieve optimal neuroprotection. Data shows very different times from ROSC to applying hypothermia, regardless if it was started by emergency medical personnel on the field or by staff in the emergency departments/intensive care units. In general, the use of induced hypothermia after CPR could be very dangerous if is not well controlled, especially if established in pre-hospital conditions. The final outcome, unfortunately, could be much more undesirable for the patient compared to the minimal improvement in neurological state.

\section{REFERENCES}

1. Nolan JP, Deakin CD, Soar J, Böttiger BW, Smith G. European Resuscitation Council Guidelines for Resuscitation 2005. Section 4. Adult advanced life support S39-86.

2. The hypothermia after cardiac arrest study group. Mild therapeutic hypothermia to improve the neurological outcome after cardiac arrest. N Eng J Med 2002;346:549-556.

3. Bernard SA, Gray TW, Buist MD, Jones BM, Silvester W, Gutteridge G, Smith K. Treatment of comatose survivors of out-of-hospital cardiac arrest with induced hypothermia. N Eng J Med 2002;346:557-563.

4. Zheng ZJ, Croft JB, Giles WH, Mensah GA. Sudden cardiac death in the United States, 1989 to 1998. Circulation 2001;104:2158-2163.

5. Nolan JP, Morley PT, Vanden Hoek TL, Hickey RW, and ALS Task Force. Therapeutic hypothermia after cardiac arrest. An advisory statement by the Advanced Life Support Task Force of the International Liaison Committee on Resuscitation. Resuscitation 2003;57:231-235.

6. Riishede L, Nielsen-Kudsk F. Myocardial effects of adrenaline, isoprenaline and dobutamine at hypothermic conditions. Pharmacol Toxicol 1990;66(5):354-360.

7. Strohmenger HU, Hemmer W, Lindner KH, Schickling J, Brown CG. Median fibrillation frequency in cardiac surgery: influence of temperature and guide to countershock therapy. Chest 1997;111:1560-1564.

8. Larach MG. Accidental hypothermia. Lancet 1995;345:490-498.

9. Lloyd EL. Accidental hypothermia. Resuscitation 1996;32:111-134

10. Delaney K. Hypothermic sudden death. In: Paradis N, Halperin HR, Nowak RM, eds. Cardiac Arrest. Baltimore, Philadelphia: Williams \& Wilkins 1996:745-760.

11. Ujhelyi MR, Sims JJ, Dubin SA, Vender J, Miller AW. Defibrillation energy requirements and electrical heterogeneity during total body hypothermia. Crit Care Med 2001;29(5):1006-1011.

12. Schwarz B, Mair P, Wagner-Berger H, Stadlbauer KH, Girg S, Wenzel V, Lindner KH. Neither vasopressin nor amiodarone improve CPR outcome in an animal model of hypothermic cardiac arrest. Acta Anaesthiol Scand 2003;47:1114-1118.

13. Kliegel A, Lorset H, Sterz F, Kliegel M, Holzer M, Uray T, Domanovits H. Cold simple intravenous infusions preceding special endovascular cooling for faster induction of mild hypothermia after cardiac arrest - a feasibility study. Resuscitation 2005;64:347-351.

14. Kudenchuk PJ, Cobb LA, Copass MK, Cummins RO, Doherty AM, Fahrenbruch CE, Hallstrom AP, Murray WA, Olsufka M, Walsh T. Amiodarone for resuscitation after out-of-hospital cardiac arrest due to ventricular fibrillation. N Engl J Med 1999;341:871-878.

15. Kudenchuk PJ. Intravenous antiarrhythmic drug therapy in the resuscitation from refractory ventricular arrhythmias. Am J Cardiol 1999;84:52R-55R.

16. Kurtz A, Sessler DI, Birnbauer F, Illievich U, Spiss C. Thermoregulatory vasoconstriction impairs active core cooling. Anesthesiology 1995:82:870-876.

17. Rajek A, Greif R, Sessler DI, Baumgardner J, Laciny S, Bastanmehr H. Core cooling by central venous infusion of ice-cold $\left(4^{\circ} \mathrm{C}\right.$ and $\left.20^{\circ} \mathrm{C}\right)$ fluid. Anesthesiology 2000;93:629-637. 